\title{
Flood Impact Assessment on Road Network and Healthcare Access at the example of Jakarta, Indonesia
}

\author{
Isabell G. Klipper ${ }^{\mathrm{a}}$, Alexander Zipf ${ }^{\mathrm{a}, \mathrm{b}}$ and Sven Lautenbach ${ }^{\mathrm{a}}$ (corresponding author) \\ isabell.klipper@gmail.com, zipf@uni-heidelberg.de, sven.lautenbach@ @eigit.org \\ ${ }^{a}$ HeiGIT at Heidelberg University, Heidelberg, Germany \\ ${ }^{\mathrm{b}}$ GIScience department, Institute of Geography, Heidelberg University, Heidelberg, Germany
}

\begin{abstract}
Climate change leads to an increasing number of flood events that poses threats to a large share of the global population. In addition to direct effects, flooding leads to indirect effect due to damages of the road infrastructure that might limit accessibility of health sites. For disaster preparedness it is important to know how flood events impact accessibility in that respect. We analyzed this at the example of the capital of Indonesia, Jakarta based on the flood event of 2013. The analysis was based on information about the road network and health sites from OpenStreetMap. We assessed impacts of the flood event by comparing centrality indicators of the road network as well as by an accessibility analysis of health sites before and during the event. The flooded areas were home to 2.75 million inhabitants and hosted 79 clinics and hospitals. The flood split the road network into several subgraphs. The city center maintained its importance for time-efficient routing as well as for easily accessible healthcare but might be prone to traffic congestion after such an event. Indirect effects via interrupted road traffic through flooded areas affected around 1.5 million inhabitants and led to an increase of travel time to the nearest hospital by five minutes based on normal traffic conditions.
\end{abstract}

Keywords. disaster preparedness, OpenStreetMap, network analysis, openrouteservice, accessibility, health sites

\section{Introduction}

Due to climate change and anthropogenic activities, the number and intensity of natural hazards has steadily increased at the global scale. Floods are the most common natural disaster worldwide, with remarkable impacts on public health and economic welfare (Alderman et al., 2012). Most low-income countries are more vulnerable to floods since they often lack resources for all phases of the disaster management cycle: preparedness, mitigation, response and recovery (Alderman et al., 2012). Cities, including their infrastructures, are vulnerable systems, and the resilience of these systems is important for maintaining their functions under shock events such as major floods. Two important components of resilience are the road network as well as the healthcare system. Access to healthcare facilities is essential for the population in their everyday life and even more in such an emergency (Luo and Wang, 2003). The analysis and evaluation of the various aspects of the road network and healthcare supply are therefore important for understanding and managing the effects of flooding.

The Indonesian capital mega-city Jakarta, located at the coast of the Java Sea, is facing a serious risk of flooding due to its physical-geographical setting: about $40 \%$ of the land is below sea level and most of the Jakarta mainland stretches across alluvial lowland with a mean elevation of $7 \mathrm{~m}$ above sea level (ADPC, 2010). The city's water systems consists of 13 rivers, drains and canals that discharge into Jakarta Bay through Jakarta's 35-km coast. In addition to flood risk by heavy rainfalls during the monsoon season, floods might be also triggered or aggrevated by storms that push sea water inland. Jakrata has faced an increasing number of flood events: the years 1996, 2002, 2007, 2013, 2014 and 2020 (Octavianti and Charles, 2019; AHA center, 2020; Lyons, 2015) were marked by intense flooding, with the year 2007 having the worst impact so far. In this year 97 deaths were counted, 500,000 people evacuated, and 60 percent of the city area flooded (Octavianti and Charles, 2019). In 2013 
14 percent of the entire city was flooded, 20 people died, and 50,000 people had to be evacuated (Octavianti and Charles, 2019). The rising sea level, land subsidence and the lack of flood protection exacerbate the situation (Baker, 2012). Due to data availability, we used the flood of 2013 as an example to analyse resilience.

A number of studies have investigated how centralness indicators could be used to assess the resilience or vulnerability of road networks to external disturbance - e.g. (e.g. Abshirini et al., 2017; Ahmadzai et al., 2019; Demšar et al., 2008; Gauthier et al., 2018; Papilloud et al., 2020; Zhang et al., 2011). Accessibility of health sites based on isochrones has been applied less frequently. Examples are an analysis for subsaharan Africa (Geldsetzer et al., 2020) as well as studies at city scale (Arrighi et al., 2019; Coles et al., 2017; Green et al., 2017; Guagliardo, 2004). In case of disasters the affected parts of the road network have to be detached from the routing graph (Eckle et al., 2016).

Combined assessment of both accessibility of health sites by means of isochrones and network connectivity in respect to resilience to flood events was seldom investigated. Since both aspects are interconnected a combined analysis might reveal aspects of concern for city planning and disaster preparedness. Our analysis aimed at:

1. identifying parts of the road system in Jakarta most critical for access to health sites in Jakarta under normal conditions and under the flood event of 2013

2. a quantification of the effect of the flood event in 2013 on access of the population to health sites.

Since OSM was the main data source of our analysis, it was furthermore necessary to assess underlying data quality aspects with respect to the fitness for purpose.

\section{Methods and Data}

The road network was extracted from OSM by overpass-turbo (Overpass-Turbo Development Team, 2020). We used the administrative city boundaries of Jakarta. The mega-city, however, does not stop at the administrative boundaries - densely populated areas continue in the surroundings. The northern islands ('Kepulauan Seribu') were excluded from the analysis, avoiding complexities such as transport by boats. The resulting area covered about $650 \mathrm{~km}^{2}$. We used recent OSM data (29/09/2020), since our analysis aimed at understanding how resilient the current city would be towards a flood event similar to the 2013 event. Completeness of OSM in the region has increased over time (c.f. figure 2), so using data from 2013 would imply the use of more incomplete OSM data.
The flood extent map for 2013 was provided by HOT Indonesia. The flood map included no information about the severity of the flooding such as water depth. Information on health sites was also provided by HOT Indonesia - the information has been incorporated by HOT Indonesia into the OSM database and is available by the usual means of accessing OSM data. In addition to the type of the health site (hospital, clinic, doctor, pharmacy), information on bed capacity was available for about $60 \%$ of the hospitals and for about $53 \%$ of the clinics. Where the information on bed capacity was missing we imputed the mean of each category.

To estimate the amount of population affected by changes in accessibility due to the flood events, WorldPop (WorldPop, 2020) data at 100m cell size resolution was used.

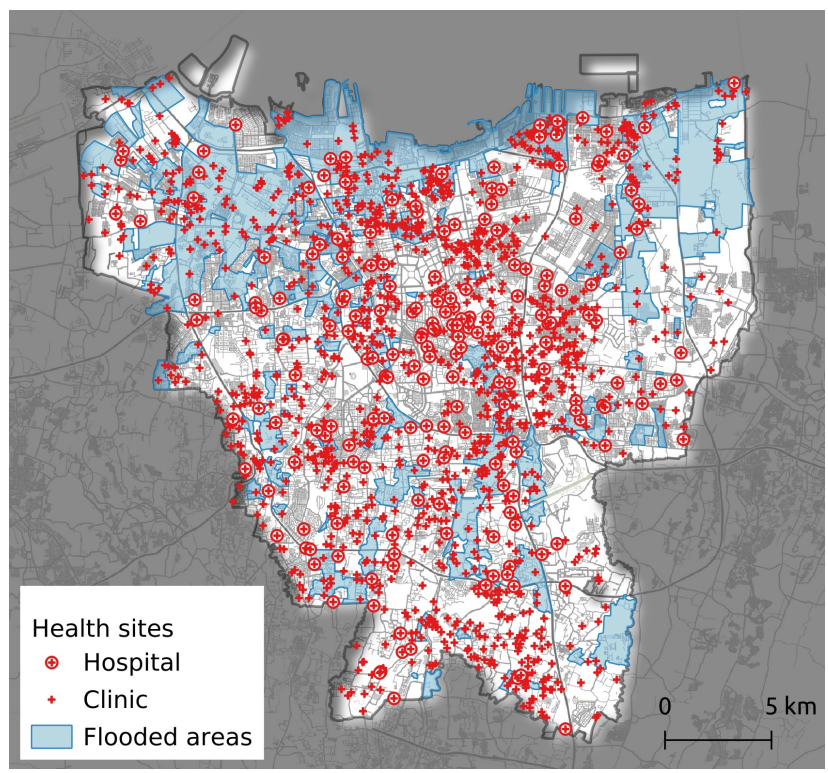

Data source: OpenStreetMap contributors, Base map: Stamen Terrain Lines, Flood layer: HOT Indonesia

Figure 1. Spatial extent of the 2013 flood and location of clinics and hospitals. Areas outside the administrative boundary of Jakarta are greyed out.

\subsection{Network analysis}

Connectivity of the road network was estimated by indicators of network centrality, namely betweenness and closeness centrality. Betweenness centrality (BC) is based on the shortest path between two nodes in the network. It measures how frequently a node lies on the shortest paths among all possible node pairs (Freeman, 1977; Wang et al., 2018). The betweenness centrality of a nodes denotes the fraction of shortest paths which pass through the focal node between all node pairs (Equation Eq. (1). The higher the betweenness centrality value of a node, the higher the importance of the node in the network. The implementation of Brandes (Brandes, 2001) was used to calculate BC. Since we 
used travel times as weights the $\mathrm{BC}$ measured in our case the frequency at which the respective nodes were approached or crossed on the fastest routes. Nodes with a high betweenness value are crossed more frequently and are in this context important for a fast routing and traffic flow. An even spatial distribution of betweenness values indicates a resilient road network, since the maintenance of traffic does not depend on individual nodes and is therefore not affected by the failure of individual sections. A doubling in value implies that the node is passed twice as often.

$$
B C(i)=\sum_{i \neq s, i \neq t, s \neq t} \frac{g_{s t}^{i}}{g_{s t}}
$$

where node $i$ is the specified node, node $s$ and $t$ are two arbitrary nodes which are distinct and also different from $i, g_{s t}$ is the number of shortest paths between $s$ and $t$, and $g_{s t}^{i}$ is the number of shortest paths via node $i$ between $s$ and $t$.

Closeness centrality (CC) (Sabidussi, 1966) counts the reciprocal of the average distance from a specific node to any other nodes within the network. Since the classic formula cannot be used for disconnected graphs we used a variant, the harmonic closeness centrality (HC, Equation 2) (Rochat, 2009). Nodes with a higher HC are closer to all other nodes and can therefor be easier reached by others (Opsahl et al., 2010). In contrast to the $\mathrm{BC}$, the results of the $\mathrm{HC}$ indicate, which nodes can be reached most quickly on average from all existing nodes. Nodes with a higher closeness rating can therefore be regarded as important supply points as well as locations which can be reached quickly by all other nodes.

$$
H C(i)=\sum_{i \neq j \in N} d_{i j}
$$

where $N$ is the set of nodes, and $d_{i j}$ denotes the distance between node $i$ and $j$.

For the analysis of connectivity only drivable roads, including residential roads were considered since we assumed transport by cars or minibuses. The analysis was performed twice: for normal conditions and for the 2013 flood event. For the flood event all roads that intersected with the flooded areas were removed from the weighted graph. Weights were based on the length of the road segment divided by the speed limit. To examine the indirect flood impact on the road network the value difference for both $\mathrm{BC}$ and $\mathrm{HC}$ was taken between the normal and the flooded scenario.

To estimate the resilience of the road network towards the flood event we calculated an adjusted version of the sameness indicator (Abshirini et al., 2017). This indicator measures how much of the existing foreground network remains unchanged after a disruption. It follows the idea that a network is less resilient, if the high centrality values do not remain with the existing nodes but are transferred to others. We used the top one percent of the centrality values as the small foreground network and the top ten percent as the large foreground network. We calculated the sameness ratio (SR) between the large foreground network for the normal and the flood event situation to quantify how strongly the flood event affected the road network. The SR ranges from 0 to 1 - higher SR values indicate that the functionality of the road network changed less.

\subsection{Accessibility analysis}

Accessibility was estimated by calculating isochrones (O'Sullivan et al., 2000; Doling, 1979) for each of the hospitals and clinics inside the administrative boundaries of Jakarta. For the flood event, only hospitals and clinics outside the flooded areas were considered. It was assumed that health sites in flooded areas were either out of order or unreachable for people in need. The openrouteservice (HeiGIT, 2020) was used to calculate the isochrones for each clinic and hospital based on the OSM road network. During pre-processing the openrouteservice builds the network graph and assigns routing weights based on OSM data. Travel time as well as traffic restrictions depend on the mode of transport. We used the car profile together with the standard speed limits of the ORS, that are assigned based on road categories if not present as tags at the individual OSM ways. The isochrones calculation considers additional factors such as driving constraints and turn restrictions if these are present in the OSM data for the region.

Isochrones were calculated for 5 minutes intervals up to half an hour of driving time. The isochrones for the two health site categories hospitals and clinics were analyzed separately. For the flood scenario flooded areas were erased from the isochrones - while flooded roads were excluded from the graph isochrones could still include smaller flooded areas under some circumstances without that extra step. The analysis was based on the simplifying assumption, that inhabitants seek help at the nearest hospital or clinic. An assumption that might hold for emergencies but not necessarily for other situations.

To estimate the relationship between the health service supplied and the demand for that service, we divided the number of beds per clinic or hospital inside each isochrone by the population in that isochrone. To examine the actual flood impact on the available health care supply, the change in the health site distribution and bed capacity was calculated by determining the difference between the results of the normal and the flooded scenario for each isochrone. 


\subsection{Intrinsic data quality assessment}

Since our analysis relies heavily on OSM data, it was necessary to check data quality. We followed an intrinsic data quality approach (Senaratne et al., 2017; Degrossi et al., 2017; Barron et al., 2014). We queried contributions for different feature classes and studied the development of contributions over time. OSM history was accessed via the ohsome API that builds on the oshdb (Raifer et al., 2019). We analyzed the development of the length of relevant highway classes, hospitals and clinics (amenity=hospital and amenity=clinic), hospitals and clinics were capacity information was present (capacity:persons $=*$ ). Definitions of the different road classes and the two health site categories can be found in the OSM Wiki (OSM Wiki, 2021a,b,c). In addition, we analyzed the number of OSM users in Jakarta that contributed actively in the domain of roads and paths (highway $\left.{ }^{*}\right)$ or amenities $($ amenity $=*)$.

\subsection{Software and Data Availability}

The code used for the analysis of network centrality and accessibility is available at github at https:/github. com/GIScience/Jakarta_Thesis_Klipper. Except the flood extent data, which were provided by the Humanitarian OpenStreetMap Team (HOT) Indonesia, all used data are publicly available.

The analysis of network centrality and accessibility was performed in Python 3.7 (Python Development Team, 2020) and QGIS (QGIS Development Team, 2020). Handling of the network data was performed by the Python OSMnx (Boeing, 2017), betweenness indicators were calculated by NetworKit (Staudt et al., 2016) and isochrones were calculated by calls to the openrouteservice API (HeiGIT, 2020) via the Python module openrouteservice 2.2.3, using the car profile and standard speed limits. In addition, the following python modules were used: fiona 1.8.13, pandas 1.0.1, numpy 1.18 .1 , shapely 1.7 .0 , geopandas 0.7 .0 , rtree 0.9.4, matplotlib 3.2.0, pyyaml 5.3, ogr2osm, rasterio 1.1.3, area 1.1.1 and rasterstats 0.14.0.

The analysis of the data quality was performed in $\mathrm{R}(\mathrm{R}$ Core Team, 2021), using the packages sf (Pebesma, 2018), RCurl (Temple Lang, 2021), geojsonio (Chamberlain and Teucher, 2021), tidyverse (Wickham et al., 2019), ggplot2 (Wickham, 2016) and ggpubr (Kassambara, 2020). R code of the analysis is available at https: //gitlab.gistools.geog.uni-heidelberg.de/giscience/ big-data/ohsome/ohsome-api-analysis-examples/ completeness_highway_healthsites_jakarta.

\section{Results}

\subsection{Data quality assessment}

The development of OSM features in Jakarta (c.f. fig. 2) shows that the length road categories relevant for car travel have been converging towards $13,000 \mathrm{~km}$. The increase in road lengths for these categories has been very small, especially since mid 2019. Additional features of the OSM key highway were mainly added for categories such as footway or path. The general development of contributions showed several phases which could be described by a multiple logistic growth function. This pattern presumably is related to several mapping campaigns triggered e.g. by HOT Indonesia. The development of highway contributions showed some shifts between different categories which might indicate a refinement of the road type classification. Visual inspection of the road network in comparison to recent high resolution imagery by Mapbox revealed also no indication of missing road segments in the case study region. Together with the sufficiently high number of active OSM contributors mapping amenities and highways (c.f. fig. 3) we can take this as an indication, that the relevant part of the road network was sufficiently complete for our analysis.

Contributions to clinics and hospitals in OSM (which are based on the HOT Indonesia data set we used) have also levelled of since 2019. A few locations of hospitals (3\% of 221$)$ and clinics $(0.5 \%$ of 1516$)$ were added since 2019 - however, we can still assume that the locations of these health sites were sufficiently complete for our analysis. As mentioned above, information about bed capacity was incomplete.

\subsection{Network analysis}

Nearly a quarter of all nodes and of the edges were affected by the flood event, which split the network into one main graph and several subgraphs. In total, 34,027 nodes were flooded and therefore no longer passable. Effects of the flood event on betweenness centrality there relatively small (cf. figure 4). A few nodes in the center of the city gained in betweenness centrality but on average the value decreased slightly from 0.00004 to 0.00002 . Most of the nodes ( 75 percent) did not change in value or only changed by a small amount of $-/+0.0005$, which means that these nodes had an increasing or decreasing traffic volume of up to 0.05 percent.

Effects of the flood event on $\mathrm{HC}$ were a bit more pronounced than for BC (cf. figure 4). More than 60 percent of the remaining 111,379 nodes changed their $\mathrm{HC}$ value by less than $-/+0.2$, which indicates that most of the nodes and the major part of the network, respectively, maintained their functionality with respect to the provision of fast access. Strongest losses in HC 


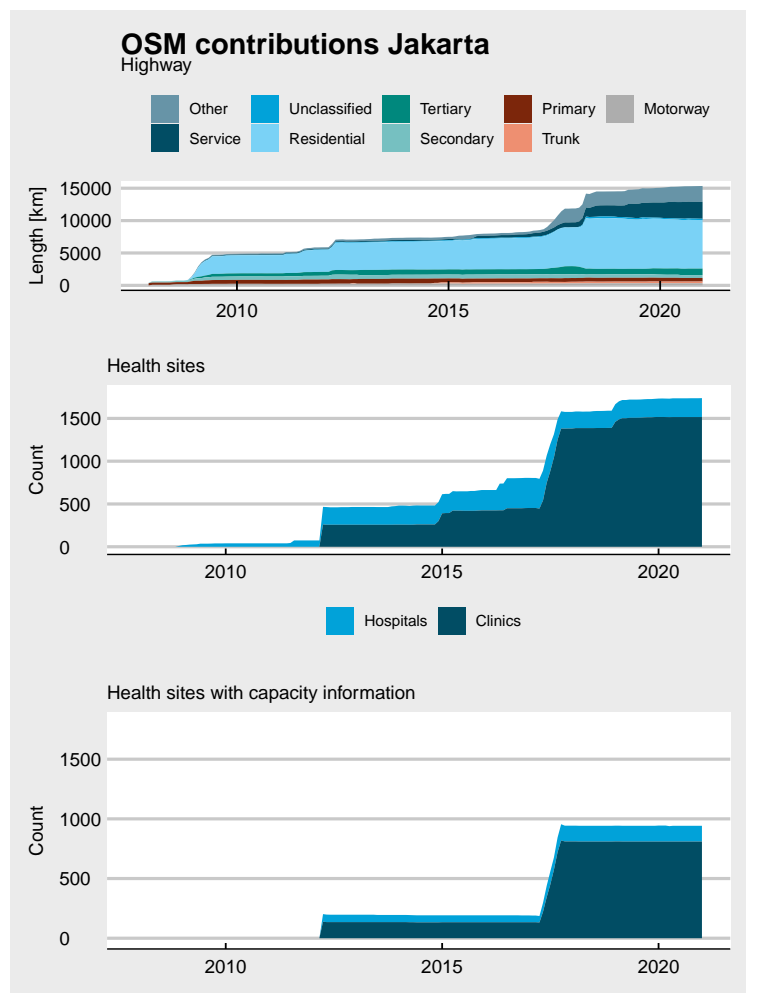

Figure 2. Intrinsic data quality assessment for relevant OSM feature classes.

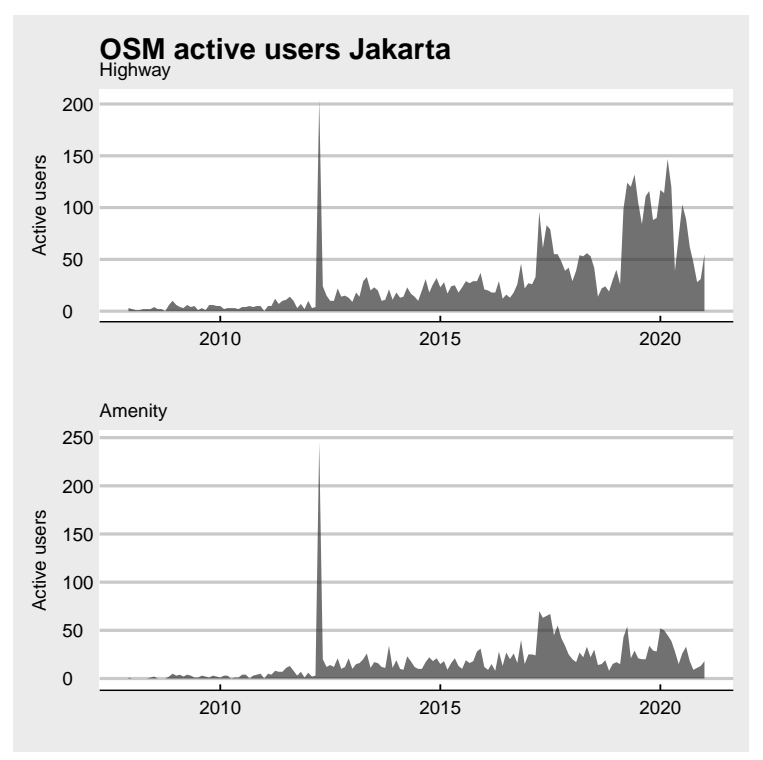

Figure 3. Intrinsic data quality assessment: number of active OSM contributors per month for relevant feature classes.

value occurred in nodes located near the city border. This might be partly due to the artificial clipping of the road network at the administrative boundary, since this limited the possibility to bypass flooded areas near the city boundary. On average the $\mathrm{HC}$ value decreased from 0.25 to 0.13 .

For the small foreground network - representing the top one percent of the $\mathrm{BC}$ values - effects on $\mathrm{BC}$ become

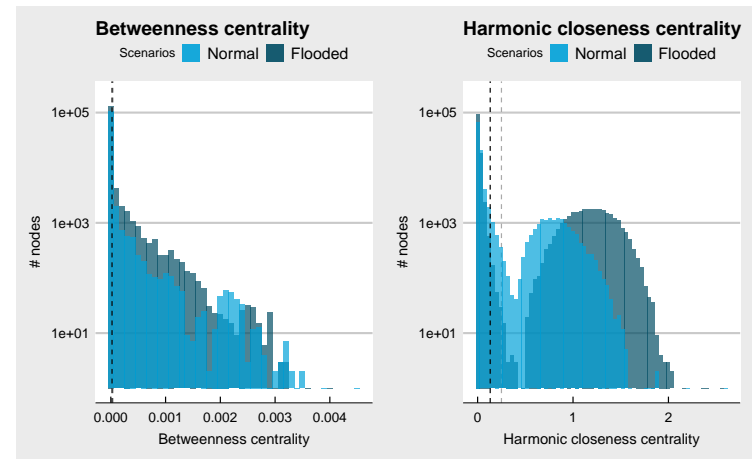

Figure 4. Histogram of $\mathrm{BC}$ and $\mathrm{HC}$ values for normal conditions and the flood event. The dashed lines represent the mean of the distribution: grey: normal, black: flooded - for betweenness centrality the two lines cannot be distinguished due to the small difference in mean.

clearer (cf. figure 5). The value of about 50 percent of these nodes decreased to a degree that indicated that their high importance within the road framework could not be maintained. Particularly affected by the flood as well as by the loss of importance, were the connecting roads to the north-west and to the south. The city centre area, on the other hand, even gained in importance in some cases. Around 4.7 percent (69 nodes) of the small foreground network increased in $\mathrm{BC}$ value $(0.0005-0.0025)$. The nodes were located particularly in the area close to the city centre on the east-west connection, running in parallel to roads which lost importance. The sameness ratio between the normal and the flooded situation was 0.62 for BC.

For the small foreground network of the top one percent $\mathrm{HC}$ values nodes concentrate mainly to the western inner-city area (cf. Fig. 5). Almost 30 percent of the nodes experienced such a strong importance decrease by the flood event, that the respective nodes were no longer part of the small foreground network. Nodes located in the centre of the city decreased in $\mathrm{HC}$ value too, but belonged still to the small foreground network - i.e. they were still of high importance for a fast supply within the city. This indicates a core maintenance and resilience of the road network for the largest sub-graph. One node located in the city center gained strongly in importance, the $\mathrm{HC}$ value changed from 0.51 to 2.18 - this node might be especially sensitive to traffic congestion. The sameness ratio between the normal and the flooded situation was 0.69 for $\mathrm{HC}$.

\subsection{Accessibility analysis}

Hospitals and clinics in Jakarta were mainly concentrated in the city center and western and southern parts of the city. The concentration of health services was further amplified since the hospitals and clinics in these locations had also the highest bed capacities. Due to the flood event, 30 (15\%) hospitals and 349 (25\%) clin- 

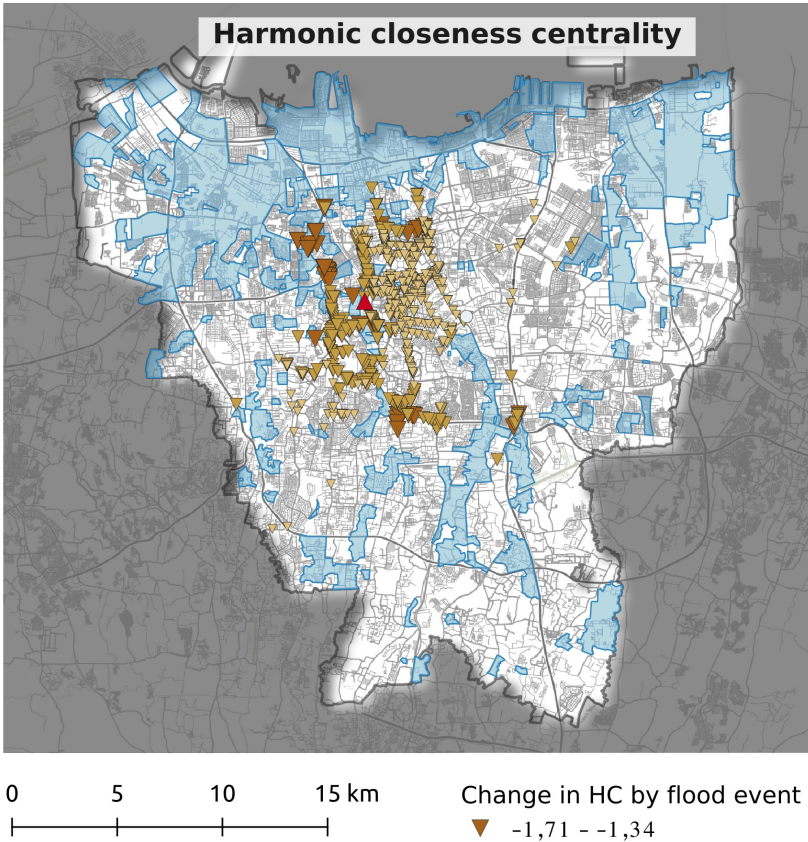

Data source: OpenStreetMap contributors Base map: Stamen Terrain Lines Flood layer: HOT Indonesia

Change in $\mathrm{HC}$ by flood event
$\begin{aligned} \nabla & -1,71--1,34 \\ \nabla & -1,34--0,5 \\ \nabla & -0,5--0,1 \\ & -0,1-0,1 \\ \boldsymbol{\Delta} & 0,1-0,51\end{aligned}$

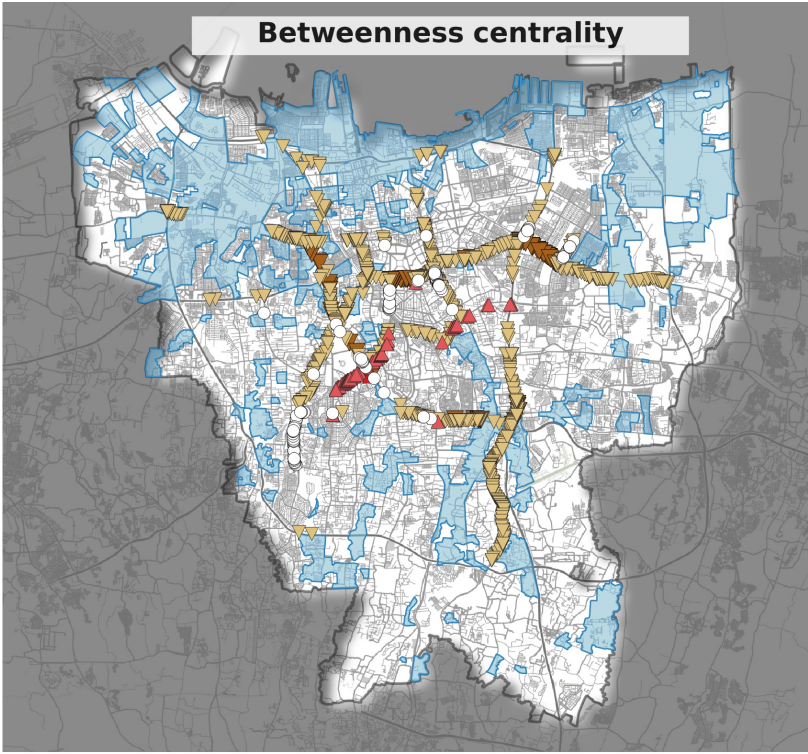

Change in $\mathrm{BC}$ by flood event $\square$ Flooded areas

$\nabla-0,0031--0,002$

$\nabla \quad-0,002--0,0005$

$-0,0005-0,0005$

$\triangle \quad 0,0005-0,002$

$0,002-0,0024$

Figure 5. Flood impact on the BC (left) and HC (right) related small foreground network and the functionality of the main road structure considering the frequency of crossed locations based on the car fastest routing profile within the administrative city borders.

ics were affected and were considered as no longer functional for our analysis. This led to a reduction of $12,000(16.6 \%)$ and $34,500(25.8 \%)$ beds in hospitals and clinics respectively.

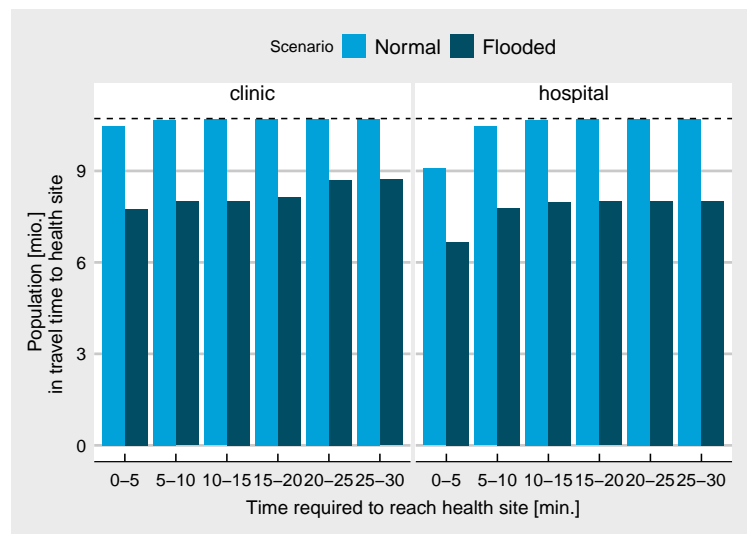

Figure 6. Amount of city population with healthcare access within specific time range: (left) access to at least one hospital, (right) access to at least one clinic. The size of the accessible area, for the normal respectively the flooded scenario, were calculated by summing up the inhabitants for the dissolved isochrones for each time interval.

Under normal conditions almost from the entire area of the city of Jakarta at least one hospital or one clinic was accessible within a 10 to 15 minute car drive (c.f. 6). Exceptions were small areas at the city border, which represent parks or areas with restricted access. From the majority of the urban area and of the urban population the closest hospital was even accessible within five minutes. The flooding had direct as well as indirect impact on accessibility to health sites. Directly affected were the flooded areas which were considered no longer passable - in total 2.75 million inhabitants ( $25.6 \%$ of the total population) lived in those areas. Indirect effects were caused by detours necessary to avoid flooded areas. These indirect impacts lead to an increase of travel time by five minutes for the inhabitants of parts of the city. In the north-western part of the city, north of the area most affected by the flood, an additional travel time of up to ten minutes occurred in some areas. Small spatial fragments close to the administrative city border were also subject to an additional travel time of more than ten minutes. In total 1.36 million inhabitants were affected by an increase in travel time to the nearest hospital from up to 5 minutes to 5 to 10 minutes and 140,000 by an increase from 5 to 10 minutes travel time to 10 to 15 minutes. With respect to the travel time to the nearest clinic the increase by indirect effects was less severe: around 60,000 inhabitants had to face an increase from up to 5 to 5 to 10 minutes.

The main roads near the city centre provided under normal conditions access up to 10,000 beds within a 5minute car drive, with bed availability in a five-minute 
drive decreasing towards the administrative boundary. The effect of the flood event on accessible to bed capacity was spatially heterogeneous (cf. Fig. 7). Especially the northern part of Jakarta was affected with a reduction of more than 0.1 beds per person per square kilometre. In the southern section, the flood caused changes of varying intensity.

\section{Discussion}

\subsection{Network analysis}

Due to the direct and indirect impacts of the 2013 flood, the road network broke up into sub-graphs. However, the major part of the network remained intact. The network experienced inevitably a reduction functionality, as road segments actively became impassable. Some non flooded areas were cut off from the main part of the city and from the main supply.

BC captured the characteristics of the road network regarding efficient and fastest possible routing between all places within the city, based on a car driving fastest routing profile. The importance of the city centre increased in case of the 2013 flooding. The flood caused a shift of the highest centrality values, which means that the usual, previously used routes were no longer the fastest connections and the traffic flow would shift to other roads. This implied a stronger traffic concentration on the main roads close to the city centre, covering a much smaller area - since the highest centrality values were spatially much closer and more compact during this flood. Such a transformation can lead to a concentration of traffic and consequently to congestion.

In comparison, $\mathrm{HC}$ identified locations that were on average fastest to reach, i.e. good supply locations within the city. These places could be used as contact points for people seeking help, but also to better coordinate the supply for the affected areas. The high proportion of nodes with a very low $\mathrm{HC}$ value, concentrated in the outskirts, revealed that access to them would become more time-consuming. The city centre and the main traffic junctions within the centre showed up in this respect. Access to the city centre was reduced in the flood scenario, indicated by the decrease in $\mathrm{HC}$ value of the nodes along the main roads, which were particularly affected by the indirect flood effects. Some parts of the main roads were no longer passable and were not able to maintain their function of providing fast supply. However, due to the large number of routing possibilities between the outer areas and the city centre, the city centre was still able to provide its main supply functions for the entire city.

\subsection{Accessibility analysis}

In terms of travel time, access to health facilities remained the same in most parts of the city, in case of the 2013 flood scenario. This is partly due to the given spatial distribution of facilities and partly due to the available choice of amenities. The areas that had to consider additional travel time were mostly located in the immediate vicinity of the flood. Accessibility for areas not affected dropped only to a relatively small amount, indicating again a relatively high resilience of the system. However, bed capacity in five-minute driving time was distributed unevenly across the city, a situation that was further aggravated by the flood event. In some cases, the flood event lead to a significant loss of supply, especially in the north-western part of the city where health supply was low already under normal conditions.

\subsection{Limitations}

Limitations of our analysis can be grouped into the following categories: 1) data quality, 2) assumptions and indicators. With respect to data quality the road network underlying the analysis could be considered satisfyingly complete. The same could be assumed for clinics and hospitals. Attribute completeness with respect to the bed capacity of clinics and hospital was suboptimal. The simple imputation by the mean might not be sufficient to draw strong conclusions. The distribution of bed capacities was negatively skewed (highest count for category $>500$ ) for hospitals and positively skewed (highest count for category $<50$ ) for hospitals. One might speculate that larger hospitals and clinics were mapped first given their importance and prominence - in this case the imputation of the mean might have resulted in too high bed capacities.

Results for accessibility of hospitals without considering the supply side were not affected by that issue. However, accessibility has been calculated using the speed limit as travel speed - this is unrealistic for traffic jams which are not atypical for a mega-city such as Jakarta. So we have to assume that accessibility is lower than suggested by our results - of course also depending on the time of the day and the weekday. Travel speed adjustments due to traffic conditions are principle possible in the openrouteservice but require realistic estimates based on observational data such as Google Directions (e.g. Keller et al. (2020)). For a major flood event one has to consider that traffic might be different from normal situation so traffic simulation models might be required. Another shortcoming of our analysis is that we estimated connectivity and accessibility by means of car transport - as much traffic in mega-cities such as Jakarta relies on public and semipublic transport such as mini-busses this presumably has also led to too high accessibility estimates. While 

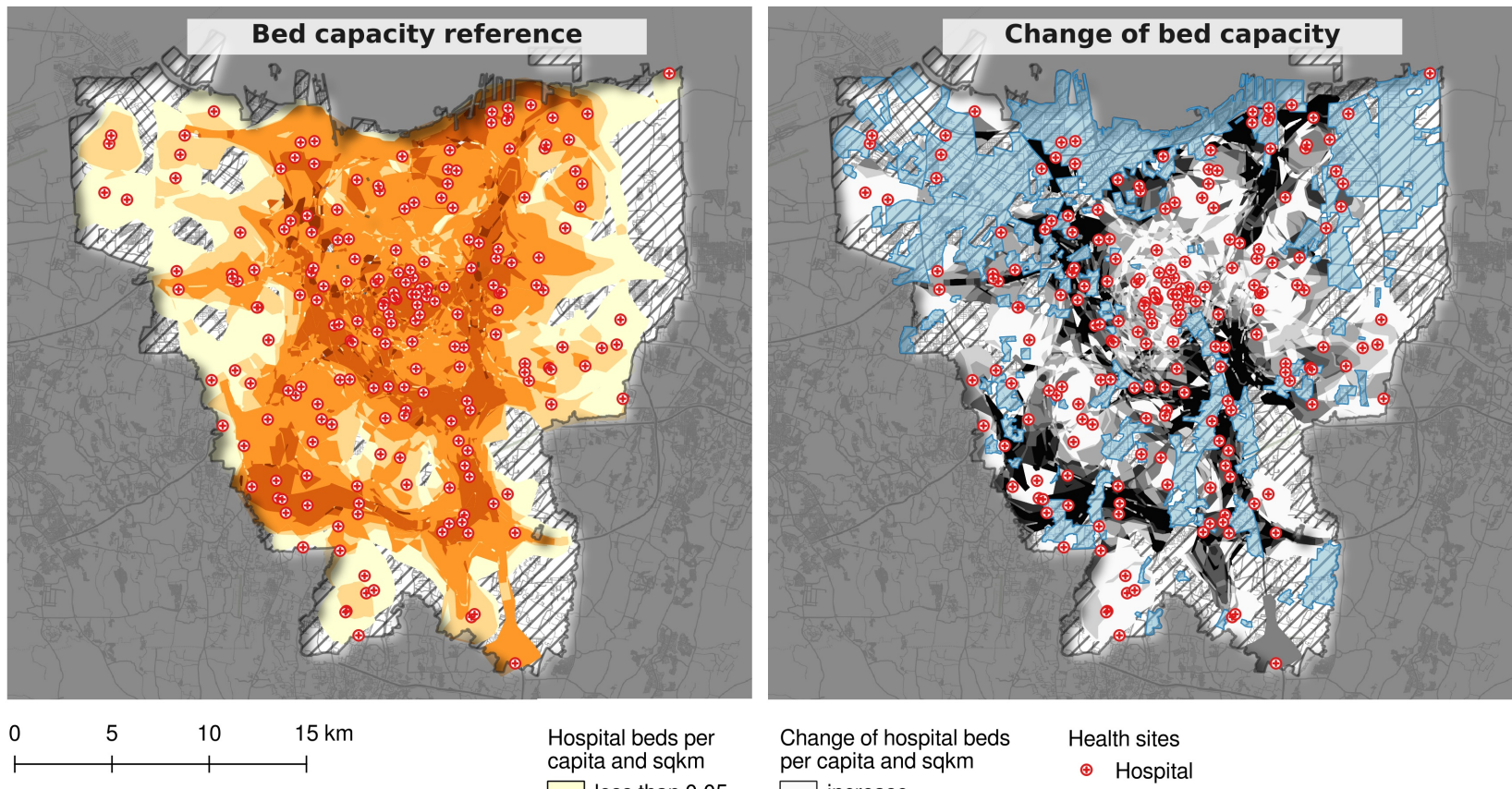

Data source: OpenStreetMap contributors Base map: Stamen Terrain Lines Flood layer and health sites: HOT Indonesia sochrones bases on openrouteservice Population by WorldPop

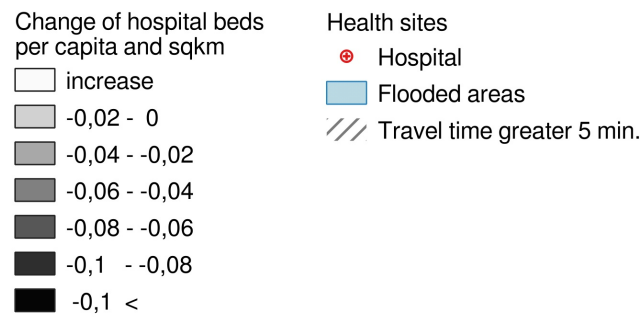

Figure 7. Cumulative amount of available hospital beds per person per square kilometre accessible within a 5-minute car drive: (left) normal scenario, (right) 2013 flood impact. Isochrone boundaries were dissolved by health site category and travel distance to generate all available isochrone fragments. The cumulative bed capacity of each fragment was set in relation to the respective population density. The flood impact was received by calculating the difference for each isochrone. Negative values indicate the loss of available beds per person per square kilometre. Since the five minutes isochrones of some hospitals and clinics decreased due to the direct and indirect effects of the flood the bed to inhabitant relationship increased in these zones relative to the normal situation since less inhabitants could reach the hospitals and clinics.

these factors require more research, our results provide still a first estimate based on freely available data. Factors influencing travel speed will presumably vary spatially, how much accessibility assessments have to take this into account - or if it is sufficient to estimate conversion factors - is up for future research.

Demand for health care was estimated simply based on population density. However, need for health care differs with age as well as with income and education. Estimating and incorporating these effects was beyond the scope of this study but should be included in upcoming work. With the focus on spatial distribution and mobility-based access to healthcare, the individual acceptance and affordability of care services was not included here. Consequently, then analysing the results, it must be kept in mind that various aspects such as gender and age, costs as well as ethnic and religious backgrounds also have an influence (Penchansky and Thomas, 1981; Jalil et al., 2018). Healthcare in Indonesia, in particular based on "out-of-pocket" financing (WHO, 2017), can lead to limited access for lower income groups. In the event of a disaster, however, re- stricted public and private care may decrease, resulting in general emergency care for all affected people. Financial resources from NGOs, international and national institutions may also help to cover the costs necessary to ensure the provision of care.

We have restricted our analysis to the administrative boundaries. However, the road network and the demand for health care extends beyond those limits. One the one hand, accessibility in some parts might be a bit higher due to the neglected possibility to bypass areas affected by flooding by taking routes outside the administrative boundaries. On the other hand, average travel times to health sites can be expected to increase significantly if considering the outskirts of Jakarta, since density of health services is clearly decreasing with increasing distance to the city center.

A further assumption we made for our analysis was that clinics and hospitals would be completely out of use if they would be flooded. Some of those places might be still functional at a reduced capacity. As long as no data is available with that respect we decided to provide conservative estimates based on the assump- 
tion that the health sites would either be not functional due to damage by the flood or that they could not be reached by emergencies and hospital/clinic staff.

\section{Conclusion and outlook}

Planning for severe disasters requires an estimation of the effects of the event on health sites and their accessibility. We have demonstrated that it is possible to address such affects by publicly available data. While information on the flood extent was not publicly available, publicly available remote sensing data such as the Sentinel products (Malenovský et al., 2012) could be used to produce this input data.

Our analysis indicated parts of the non-flooded road network that would decrease in importance and parts that would increase in importance. If roads gaining in importance are not designed for increased travel this might lead to traffic congestion with negative effects on accessibility on health sites. With respect to accessibility of clinics and hospitals we were able to pinpoint areas what would suffer most from a similar event.

While our analysis captures only the 2013 flood event, it can be assumed that affected areas would be similar for other flood events. Flooded areas during the 1996, 2002 and 2007 event showed very similar pattern to the 2013 flood event (c.f. ADPC, 2010; Texier, 2008) - therefore, our analysis of the 2013 flood event should provide important insights for city administration, health services and NGOs. While the effect of climate change on flood risk is highly uncertain (Budiyono et al., 2016), raising sea level and land subsidence are likely to increase flood risk (Budiyono et al., 2016; Takagi et al., 2016) and damage (Fajar Januriyadi et al., 2018) in Jakarta. Disaster preparedness is therefore of high importance. Our results highlight that not only the directly affected areas but also indirect effects that increase pressure on the health system should be considered in spatial planning. While OSM data quality is varying between regions and should always be carefully checked, we assume that our approach can be transferred to other mega-cities and urban areas and help to prepare for upcoming disasters.

Future work could rerun the analysis for different historic flood events or flood simulations to study the relationship between severity of the flood event and the effects on the road network and accessibility of health centers. Longer term goal is the establishment of a web-service to allow city administrations, emergency agencies and NGOs to estimate the vulnerability of urban systems - specifically with regard to health care accessibility - by floods and other natural disasters.

Acknowledgements. We would like to thank HOT Indonesia as well as the Jakarta Disaster Management Agency for pro- viding as access to the flood map data as well as to the most recent version of the health sites data base. Furthermore we would like to thank the GIScience Heidelberg team members and the HeiGIT Smart Location-based Services and Navigation, Big Spatial Data Analytics and Geoinformation for Humanitarian Aid teams for fruitful discussions and support.

\section{References}

Abshirini, E., Koch, D., and Legeby, A.: Flood hazard and its impact on the resilience of cities: An accessibility-based approach to amenities in the city of Gothenburg, Sweden, in: 11th International Space Syntax Symposium, SSS 2017, Lisbon, Portugal, 3 July 2017 through 7 July 2017, pp. 36-1, 2017.

ADPC: Flood preparedness initiatives of highrisk communities of Jakarta, Tech. Rep. 27, Asian Disaster Preparedness Center, Bangkok, Thailand, http://www.adpc.net/igo/category/ID226/doc/ 2013-c28Jbn-ADPC-Safer_Cities_27.pdf, 2010.

AHA center: Situation Updates No. 3: Massive Floods in Greater Jakarta Area - Indonesia., Tech. rep., AHA center, https://reliefweb.int/sites/reliefweb.int/files/resources/AHA Situation_Updates_No3_Greater_Jakarta_Floods_FINAL. pdf, 2020.

Ahmadzai, F., Rao, K., and Ulfat, S.: Assessment and modelling of urban road networks using Integrated Graph of Natural Road Network (a GIS-based approach), Journal of Urban Management, 8, 109-125, https://doi.org/10.1016/j.jum.2018.11.001, 2019.

Alderman, K., Turner, L. R., and Tong, S.: Floods and human health: A systematic review, Environment International, 47, 37-47, https://doi.org/10.1016/j.envint.2012.06.003, 2012.

Arrighi, C., Pregnolato, M., Dawson, R., and Castelli, F.: Preparedness against mobility disruption by floods, Science of The Total Environment, 654, 1010-1022, https://doi.org/10.1016/j.scitotenv.2018.11.191, 2019.

Baker, J. L.: Climate change, disaster risk, and the urban poor: cities building resilience for a changing world, The World Bank, 2012.

Barron, C., Neis, P., and Zipf, A.: A Comprehensive Framework for Intrinsic OpenStreetMap Quality Analysis, Transactions in GIS, 18, 877-895, https://doi.org/10.1111/tgis.12073, 2014.

Boeing, G.: OSMnx: New methods for acquiring, constructing, analyzing, and visualizing complex street networks, Computers, Environment and Urban Systems, 65, 126-139, https://doi.org/10.1016/j.compenvurbsys.2017.05.004, 2017.

Brandes, U.: A Faster Algorithm for Betweenness Centrality, Journal of Mathematical Sociology, 25, 163-177, 2001.

Budiyono, Y., Aerts, J. C. J. H., Tollenaar, D., and Ward, P. J.: River flood risk in Jakarta under scenarios of future change, Natural Hazards and Earth System Sciences, 16, 757-774, https://doi.org/10.5194/nhess-16-757-2016, 2016.

Chamberlain, S. and Teucher, A.: geojsonio: Convert Data from and to 'GeoJSON' or 'TopoJSON', https://CRAN $\mathrm{R}$-project.org/package=geojsonio, $\mathrm{r}$ package version 0.9 .4 , 2021. 
Coles, D., Yu, D., Wilby, R. L., Green, D., and Herring, Z.: Beyond 'flood hotspots': Modelling emergency service accessibility during flooding in York, UK, Journal of Hydrology, 546, 419-436, https://doi.org/10.1016/j.jhydrol.2016.12.013, 2017.

Degrossi, L. C., Albuquerque, J. P. d., Rocha, R. d. S., and Zipf, A.: A Framework of Quality Assessment Methods for Crowdsourced Geographic Information : a Systematic Literature Review, in: Proceedings of the 14th ISCRAM Conference, pp. 21-24, issue: May, 2017.

Demšar, U., Špatenková, O., and Virrantaus, K.: Identifying Critical Locations in a Spatial Network with Graph Theory, Transactions in GIS, 12, 61-82, https://doi.org/10.1111/j.1467-9671.2008.01086.x, 2008.

Doling, J.: Accessibility and strategic planning, Tech. Rep. Research Memo 75 Monograph, Birmingham: Centre for Urban and Regional Studies, University of Birmingham, 1979.

Eckle, M., de Albuquerque, J. P., Herfort, B., Zipf, A., Leiner, R., Wolff, R., and Jacobs, C.: Leveraging OpenStreetMap to Support Flood Risk Management in Municipalities: A Prototype Decision Support System., in: ISCRAM, 2016.

Fajar Januriyadi, N., Kazama, S., Riyando Moe, I., and Kure, S.: Evaluation of future flood risk in Asian megacities: a case study of Jakarta, Hydrological Research Letters, 12, 14-22, https://doi.org/10.3178/hrl.12.14, 2018.

Freeman, L. C.: A set of measures of centrality based on betweenness, Sociometry, pp. 35-41, 1977.

Gauthier, P., Furno, A., and Faouzi, N.-E. E.: Road Network Resilience: How to Identify Critical Links Subject to Dayto-Day Disruptions, Transportation Research Record, 2672, 54-65, https://doi.org/10.1177/0361198118792115, 2018.

Geldsetzer, P., Reinmuth, M., Ouma, P. O., Lautenbach, S., Okiro, E. A., Bärnighausen, T., and Zipf, A.: Mapping physical access to health care for older adults in subSaharan Africa and implications for the COVID-19 response: a cross-sectional analysis, The Lancet Healthy Longevity, 1, e32-e42, https://doi.org/https://doi.org/10.1016/S26667568(20)30010-6, 2020.

Green, D., Yu, D., Pattison, I., Wilby, R., Bosher, L., Patel, R., Thompson, P., Trowell, K., Draycon, J., Halse, M., Yang, L., and Ryley, T.: City-scale accessibility of emergency responders operating during flood events, Natural Hazards and Earth System Sciences, 17, 1-16, https://doi.org/10.5194/nhess-17-1-2017, 2017.

Guagliardo, M. F.: Spatial accessibility of primary care: concepts, methods and challenges, International Journal of Health Geographics, p. 13, 2004.

HeiGIT: openrouteservice, https://openrouteservice.org, [Online; accessed 29-September-2020], 2020.

Jalil, I. A., Rasam, A. R. A., Adnan, N. A., Saraf, N. M., and Idris, A. N.: Geospatial network analysis for healthcare facilities accessibility in semi-urban areas, in: 2018 IEEE 14th International Colloquium on Signal Processing Its Applications (CSPA), pp. 255-260, https://doi.org/10.1109/CSPA.2018.8368722, 2018.

Kassambara, A.: ggpubr: 'ggplot2' Based Publication Ready Plots, https://CRAN.R-project.org/package=ggpubr, r package version $0.4 .0,2020$.
Keller, S., Gabriel, R., and Guth, J.: Machine Learning Framework for the Estimation of Average Speed in Rural Road Networks with OpenStreetMap Data, ISPRS International Journal of Geo-Information, 9, 638, https://doi.org/10.3390/ijgi9110638, 2020.

Luo, W. and Wang, F.: Measures of Spatial Accessibility to Health Care in a GIS Environment: Synthesis and a Case Study in the Chicago Region, Environment and Planning B: Planning and Design, 30, 865-884, https://doi.org/10.1068/b29120, 2003.

Lyons, S.: The Jakarta floods of early 2014: Rising risks in one of the World's fastest sinking cities, Tech. rep., Liège Université SEM, http://labos. ulg.ac.be/hugo/wp-content/uploads/sites/38/2017/11/ The-State-of-Environmental-Migration-2015-103-120.pdf, 2015

Malenovský, Z., Rott, H., Cihlar, J., Schaepman, M. E., García-Santos, G., Fernandes, R., and Berger, M.: Sentinels for science: Potential of Sentinel-1, -2, and -3 missions for scientific observations of ocean, cryosphere, and land, Remote Sensing of Environment, 120, 91-101, https://doi.org/10.1016/j.rse.2011.09.026, iSBN: 0034-4257, 2012.

Octavianti, T. and Charles, K.: The evolution of Jakarta's flood policy over the past 400 years: The lock-in of infrastructural solutions, Environment and Planning C: Politics and Space, 37, 1102-1125, https://doi.org/10.1177/2399654418813578, 2019.

Opsahl, T., Agneessens, F., and Skvoretz, J.: Node centrality in weighted networks: Generalizing degree and shortest paths, Social Networks, 32, 245-251, https://doi.org/10.1016/j.socnet.2010.03.006, 2010.

OSM Wiki: OpenStreetMap Wiki tag:amenity=clinic, https: //wiki.openstreetmap.org/wiki/Tag:amenity\%3Dclinic/, [Online; accessed 28-April-2021], 2021a.

OSM Wiki: OpenStreetMap Wiki key:highway, https: //wiki.openstreetmap.org/wiki/Key:highway/, [Online; accessed 28-April-2021], 2021b.

OSM Wiki: OpenStreetMap Wiki tag:amenity=hospital, https://wiki.openstreetmap.org/wiki/Tag:amenity\% 3Dhospital/, [Online; accessed 28-April-2021], 2021c.

O'Sullivan, D., Morrison, A., and Shearer, J.: Using desktop GIS for the investigation of accessibility by public transport: an isochrone approach, International Journal of Geographical Information Science, 14, 85-104, https://doi.org/10.1080/136588100240976, 2000.

Overpass-Turbo Development Team: Overpass-Turbo, https: //overpass-turbo.eu/, [Online; accessed 10-June-2020], 2020.

Papilloud, T., Röthlisberger, V., Loreti, S., and Keiler, M.: Flood exposure analysis of road infrastructure Comparison of different methods at national level, International Journal of Disaster Risk Reduction, 47, 101548 , https://doi.org/10.1016/j.ijdrr.2020.101548, https://www. sciencedirect.com/science/article/pii/S221242091931413X, 2020.

Pebesma, E.: Simple Features for R: Standardized Support for Spatial Vector Data, The R Journal, 10, 439-446, https://doi.org/10.32614/RJ-2018-009, 2018. 
Penchansky, R. and Thomas, J. W.: The Concept of Access: Definition and Relationship to Consumer Satisfaction, Medical Care, 19, 127-140, https://doi.org/10.1097/00005650198102000-00001, 1981.

Python Development Team: Python, https://www.python. org/, [Online; accessed 10-June-2020], 2020.

QGIS Development Team: QGIS Geographic Information System, Open Source Geospatial Foundation, http://qgis.org, 2020.

R Core Team: R: A Language and Environment for Statistical Computing, R Foundation for Statistical Computing, Vienna, Austria, https://www.R-project.org/, 2021.

Raifer, M., Troilo, R., Kowatsch, F., Auer, M., Loos, L., Marx, S., Przybill, K., Fendrich, S., Mocnik, F.-B., and Zipf, A.: OSHDB: a framework for spatio-temporal analysis of OpenStreetMap history data, Open Geospatial Data, Software and Standards, 4, https://doi.org/10.1186/s40965-0190061-3, 2019.

Rochat, Y.: Closeness centrality extended to unconnected graphs: The harmonic centrality index, in: ASNA, EPFLCONF-200525, 2009.

Sabidussi, G.: The centrality index of a graph, Psychometrika, 31, 581-603, 1966.

Senaratne, H., Mobasheri, A., Ali, A. L., Capineri, C., and Haklay, M. M.: A review of volunteered geographic information quality assessment methods, International Journal of Geographical Information Science, 31, 139167, https://doi.org/10.1080/13658816.2016.1189556, https://www.tandfonline.com/doi/full/10.1080/13658816. 2016.1189556, 2017

Staudt, C. L., Sazonovs, A., and Meyerhenke, H.: NetworKit: A tool suite for large-scale complex network analysis, Network Science, 4, 508-530, 2016.

Takagi, H., Esteban, M., Mikami, T., and Fujii, D.: Projection of coastal floods in 2050 Jakarta, Urban Climate, 17, 135145, https://doi.org/10.1016/j.uclim.2016.05.003, 2016.

Temple Lang, D.: RCurl: General Network (HTTP/FTP/...) Client Interface for R, https://CRAN.R-project.org/package= RCurl, r package version 1.98-1.3, 2021.

Texier, P.: Floods in Jakarta: when the extreme reveals daily structural constraints and mismanagement, Disaster Prevention and Management: An International Journal, 17, 358-372, https://doi.org/10.1108/09653560810887284, https://www.emerald.com/insight/content/doi/10.1108/ 09653560810887284/full/html, 2008.

Wang, J., Li, C., and Xia, C.: Improved centrality indicators to characterize the nodal spreading capability in complex networks, Applied Mathematics and Computation, 334, 388-400, https://doi.org/10.1016/j.amc.2018.04.028, 2018.

WHO: The Republic of Indonesia Health System Review, Tech. rep., World Health Organization, https://apps.who.int/iris/bitstream/handle/10665/254716/ 9789290225164-eng.pdf;sequence=1., 2017.

Wickham, H.: ggplot2: Elegant Graphics for Data Analysis, Springer-Verlag New York, https://ggplot2.tidyverse.org, 2016.

Wickham, H., Averick, M., Bryan, J., Chang, W., McGowan, L. D., François, R., Grolemund, G., Hayes, A., Henry, L.,
Hester, J., Kuhn, M., Pedersen, T. L., Miller, E., Bache, S. M., Müller, K., Ooms, J., Robinson, D., Seidel, D. P., Spinu, V., Takahashi, K., Vaughan, D., Wilke, C., Woo, K., and Yutani, H.: Welcome to the tidyverse, Journal of Open Source Software, 4, 1686, https://doi.org/10.21105/joss.01686, 2019.

WorldPop: Indonesia - Population Counts, https://data.humdata.org/dataset/ worldpop-population-counts-for-indonesia, [Online; accessed 29-September-2020], 2020.

Zhang, Y., Wang, X., Zeng, P., and Chen, X.: Centrality Characteristics of Road Network Patterns of Traffic Analysis Zones, Transportation Research Record: Journal of the Transportation Research Board, 2256, 16-24, https://doi.org/10.3141/2256-03, 2011. 